\title{
Filigrane
}

Écoutes psychothérapiques

\section{Traumatisme, solitude et auto-engendrement. Annie Ernaux : L’événement}

\section{Georges Gaillard}

Volume 15, numéro 1, printemps 2006

URI : https://id.erudit.org/iderudit/013530ar

DOI : https://doi.org/10.7202/013530ar

Aller au sommaire du numéro

\section{Éditeur(s)}

Revue Santé mentale au Québec

\section{ISSN}

1192-1412 (imprimé)

1911-4656 (numérique)

Découvrir la revue

Citer cet article

Gaillard, G. (2006). Traumatisme, solitude et auto-engendrement. Annie

Ernaux : L'événement. Filigrane, 15(1), 67-86. https://doi.org/10.7202/013530ar

\section{Résumé de l'article}

Au cours de la période de remaniement identificatoire du passage à l'âge adulte, la confrontation au traumatisme, et les vécus de transgressions opèrent, pour le Je, comme une tentative de séparation d'avec la psyché maternelle en ses points d'aliénation. Lorsque ces temps sont vécus dans la solitude, et ne sont pas socialisés par un lien au groupe, les éprouvés traumatiques sont configurés sur le mode de l'auto-fondation. Le sujet court alors le risque de se désarrimer de la communauté humaine sous le couvert de la revendication identitaire d'un statut d'exception. Ces configurations sont dépliées en appui sur un ouvrage de l'écrivain Annie Ernaux. Son texte autobiographique L'événement (2000), vaut comme paradigme de ces vécus traumatiques éprouvés par le Je dans la solitude, au seuil de l'âge adulte. Il permet de lire avec précision le mouvement de revendication identitaire et d'auto-engendrement mis en jeu à l'endroit même du traumatisme.
Ce document est protégé par la loi sur le droit d'auteur. L'utilisation des services d'Érudit (y compris la reproduction) est assujettie à sa politique d'utilisation que vous pouvez consulter en ligne.

https://apropos.erudit.org/fr/usagers/politique-dutilisation/ 


\title{
Traumatisme, solitude et auto- engendrement. Annie Ernaux: L'événement
}

\author{
georges gaillard
}

\begin{abstract}
Au cours de la période de remaniement identificatoire du passage à l'âge adulte, la confrontation au traumatisme, et les vécus de transgressions opèrent, pour le $\mathrm{Je}$, comme une tentative de séparation d'avec la psyché maternelle en ses points d'aliénation. Lorsque ces temps sont vécus dans la solitude, et ne sont pas socialisés par un lien au groupe, les éprouvés traumatiques sont configurés sur le mode de l'autofondation. Le sujet court alors le risque de se désarrimer de la communauté humaine sous le couvert de la revendication identitaire d'un statut d'exception. Ces configurations sont dépliées en appui sur un ouvrage de l'écrivain Annie Ernaux. Son texte autobiographique L'événement (2000), vaut comme paradigme de ces vécus traumatiques éprouvés par le Je dans la solitude, au seuil de l'âge adulte. Il permet de lire avec précision le mouvement de revendication identitaire et d'auto-engendrement mis en jeu à l'endroit même du traumatisme.
\end{abstract}

«J'ai fini de mettre en mots ce qui m'apparaît comme une expérience humaine totale, de la vie et de la mort, du temps, de la morale et, de l'interdit, de la loi, une expérience vécue d'un bout à l'autre au travers du corps.» (A. Ernaux $2000^{1}$ ).

«C'est pourquoi l'on peut dire que le savoir, fût-il le plus sublimé, est ce qui permet au sujet d'accepter l'inacceptable de la castration, en plaçant l'enjeu de son savoir en ce point précis où transgression et assomption engagent une partie jamais close.» (P. Aulagnier 1967²).

$A$ u cours de la période de remaniement identificatoire du passage à l'âge adulte, la confrontation au traumatisme, agie/vécue sur un mode transgressif, opère pour le Je comme une (nouvelle) tentative de séparation d'avec la psyché maternelle en ses points d'indifférenciation aliénants. L'ensemble des rituels initiatiques par où se marque ce même passage dans des sociétés dites «traditionnelles» relate communément le recours au traumatisme, mis au service d'une séparation, voulue comme radicale, entre le monde de l'enfance, et le nouveau rôle social, d'homme ou de femme, visé. Une nouvelle temporalité, une nouvelle identité, sont définies à partir de ces moments «initiatiques ». Le savoir sur la vie, la mort, et la sexualité (adulte) font partie des « révélations » qui caractérisent ces initiations. Si le traumatisme vise une rupture, une transformation des modalités de lien du sujet à ses proches et au groupe social, il vise conjointement à agréger le sujet à son nouveau groupe d'appartenance, et à promouvoir (en urgence) de nouvelles identifications ${ }^{3}$. Lorsque ces temps charnières sont vécus par le Je dans la solitude, lorsqu'ils ne sont pas mis en partage, et ne sont pas 
médiatisés, socialisés, par l'intermédiaire d'un groupe, les éprouvés traumatiques sont configurés sur le mode de l'auto-fondation. L'expérience vaut alors non pour son contenu, mais pour son effet effractif.

Il importe que ces expériences gardent leur potentiel d'étrangeté et de dérangement, de manière à assurer le Je qu'il y a bien en lui de l'étranger — de l'étranger au discours énoncé par la psyché parentale/maternelle, mis en place de discours unique. Il s'agit ainsi de fabriquer de l'étrangeté, pour échapper à l'envahissement de l'autre (étranger-familier) en soi. Le sujet court alors le risque de se désarrimer de la communauté humaine sous le couvert de la revendication identitaire d'un statut d'exception.

Pour déplier ces configurations, nous allons avoir recours à la littérature, et nous référer à un ouvrage de l'écrivain contemporain Annie Ernaux. Un de ses textes (2000) s'intitule L'événement; il relate une expérience autobiographique vécue durant ces temps de remaniements identitaires de l'entrée dans l'âge adulte. Au travers de son écrit, l'auteure nous permet de lire avec précision le mouvement de revendication identitaire mis en jeu à l'endroit même du traumatisme. S'y dessine avec netteté le nouage entre éprouvés traumatiques, paralysie de la pensée et assimilation à une position héroïque. Nous allons donc octroyer à ce témoignage le statut de paradigme de ces vécus traumatiques éprouvés par le Je dans la solitude, au seuil de l'âge adulte. Ce mode d'appropriation de sa propre subjectivité, qui procède de la transgression, se rencontre d'autant plus massivement que le Je se trouve aux prises avec des processus d'empiétements de la psyché maternelle et/ou parentale sur sa propre psyché.

$\mathrm{Au}$ travers de la reprise du contenu et de la forme du récit L'événement, nous allons nous attacher à dégager le statut psychique que l'écrivain Annie Ernaux a conféré à cette expérience «exceptionnelle », la manière dont elle s'y est configurée. Ceci nous permettra de voir œuvrer les mouvements de liaison — déliaison en jeu. L'accent sera mis sur les enfermements paradoxaux dans lequel le Je se précipite, sous le couvert d'une visée de désaliénation de l'emprise de la psyché de l'autre maternel. Au cours de la traversée de ces expériences le Je s'arrime à ses éprouvés; il leur octroie alors le pouvoir de le ré-originer en un mouvement d'auto-fondation, d'auto-engendrement.

\section{L'engluement dans une expérience «extrême»}

L'événement: le titre de l'ouvrage d'Annie Ernaux indique dès l'abord l'importance conférée par l'auteure à ce qui va être relaté. Si cette écrivain est connue pour ses récits autobiographiques à l'écriture factuelle et acérée, et si cet événement-là mérite d'être appelé l' «événement», on se trouve donc devant une situation carrefour, un moment charnière, où s'est joué pour le Je, une importante bascule dans ses identifications. Le singulier distingue cet «événement»de l'ensemble des évènements vécus. Le statut psychique que revêt pour le Je cette situation est donc indiqué d'emblée, dans la manière même dont l'auteure se place face à l'expérience, lui octroyant un statut d'exception. 
En quatrième de couverture, le lecteur accède à un court extrait du texte, qui annonce ce que l'ouvrage va engager:

\begin{abstract}
«Depuis des années je tourne autour de cet événement de ma vie. Lire dans un roman le récit d'un avortement me plonge dans un saisissement sans images ni pensées, comme si les mots se changeaient instantanément en sensation violente. De la même façon entendre par hasard La javanaise. J'ai la mémoire qui flanche, n'importe quelle chanson qui m'a accompagnée durant cette période me bouleverse. »
\end{abstract}

Il est donc question d'une expérience et d'un récit d'avortement. Selon ces quelques lignes proposées à la lecture sans avoir à s'aventurer entre les pages, Annie Ernaux, donne à entendre qu'il y a du traumatisme, du bouleversement.

Avec cette expérience, on se trouve en présence d'expériences que le Je maintient hors liaison d'une chaîne signifiante, expériences auxquelles toutefois il s'assimile, leur attribuant la vertu de lui octroyer un statut hérö̈que. Elles mettent dès lors le Je dans une solitude souffrante, où masochisme et jouissance sont à l'œuvre. Le Je préserve de telles expériences en ce qu'elles lui confèrent un statut d'exception, et qu'elles lui permettent (au prix du traumatisme et de la souffrance corrélée) de s'extraire de l'engluement maternel (et parental). A. Ernaux indique clairement que cette expérience n'a fait l'objet d'aucune métabolisation avant cette mise en écriture: les mots qui pourraient témoigner de l'expérience auprès d'un autre et humaniser ce trouble par/dans le lien, ces mots sont immédiatement rabattus dans une sensorialité violente, sous le couvert de «l'indicible». Les images sont exclues en même temps que les pensées; seul demeure le saisissement.

\begin{abstract}
«Les mois qui ont suivi baignent dans une lumière de limbes. Je me vois dans les rues en train de marcher continuellement. À chaque fois que j'ai pensé à cette période, il m'est venu en tête des expressions littéraires telles que «la traversée des apparences », «par-delà le bien et le mal» ou encore «le voyage au bout de la nuit». Cela m'a toujours paru correspondre à ce que j'ai vécu et éprouvé alors, quelque chose d'indicible et d'une certaine beauté. » (p. 23-24).
\end{abstract}

Le manuscrit de L'événement est daté de 1999; l'expérience traumatique s'est, elle, déroulée en 1963. A. Ernaux reprend donc 36 ans plus tard, un vécu qui reste présent dans toute sa charge émotionnelle et sensorielle.

\title{
Le suspens du temps et le désarrimage du Je de la chaîne historisante
}

Dans l'expérience traumatique relatée, le rapport au temps est altéré : le Je cesse d'investir le temps futur, et tente, (en s'y perdant), de placer l'expérience dans un 
hors temps qui résonne avec le temps du rêve. Lors de l'écriture, Annie Ernaux sera ainsi ressaisie dans cet épaississement («ce temps qui s'épaissi[ssai]t sans avancer»), à l'identique avec ce qui s'est altéré des années auparavant. Où plutôt, elle recherchera activement l'actualisation de cet épaississement.

«(Je sens que le récit m'entraîne et impose, à mon insu, un sens, celui du malheur en marche inéluctablement. Je m'oblige à résister au désir de dévaler les jours et les semaines, tâchant de conserver par tous les moyens - la recherche et la notation des détails, l'emploi de l'imparfait, l'analyse des faits — l'interminable lenteur d'un temps qui s'épaississait sans avancer, comme celui des rêves.)» (p. 44).

Lors du vécu de l'expérience, c'est l'investissement du temps futur qui s'est trouvé altéré, interdit. Investir le temps, dans son écoulement, équivalait alors à accepter la réalité de l'état de grossesse. Or Annie Ernaux témoigne comment toute son énergie a été précisément mobilisée autour d'un refus, d'une négation, celui de cet état de grossesse. Elle s'est employée à arrêter magiquement le temps, à le nier. Le projet d'avortement ne lui permet pas cet investissement du futur, si ce n'est sous la forme de l'immédiateté d'une volonté destructrice «que cela cesse ». S'inscrire alors dans la temporalité ç'eût été pouvoir parler avec d'autres, et dans une mise en représentation, confronter les questions auxquelles cette expérience conviait: considérer le corps dans sa féminité et sa maternité révélée, interroger la sexualité et les liens qu'elle présuppose avec le désir et la configuration œdipienne, interroger la volonté de mort à l'œuvre. À la place de ces confrontations, le Je perd ses repères, devient informe, analogue à ce temps refusé, qui ne peut que s' «épaissir», «devenir une chose informe».

«Le temps a cessé d'être une suite insensible de jours à remplir de cours, d'exposés, de stations dans les cafés et à la bibliothèque, menant aux examens et aux vacances d'été, à l'avenir. Il est devenu une chose informe qui avançait à l'intérieur de moi et qu'il fallait détruire à tout prix.» (p. 28).

Le déni de la temporalité convoque une toute puissance imaginaire, sans qu'Annie Ernaux ne rencontre aucun «autre», en mesure de faire pièce à ce vertige. La pensée est alors investie en tant que puissance de destruction, en tant que force d'emprise sur le corps du sujet, au service de la déliaison. S'autoriser à penser équivalait à donner une réalité au fotus, soit précisément donner consistance à ce qui est refusé: que «cette chose là » soit.

«Pour penser ma situation, je n'employais aucun des termes qui la désignent, ni "enceinte", encore moins "grossesse", voisin de 
“grotesque". Ils contenaient l'acceptation d'un futur qui n'aurait pas lieu. Ce n'était pas la peine de nommer ce que j'avais décidé de faire disparaître. Dans l'agenda j'écrivais : "ça", "cette choselà", une seule fois "enceinte”.» (p. 28).

Or c'est ce mouvement même de négation, d'effacement de cette «chose-là », qui altère l'équilibre du Je. S'engage ainsi une lutte pour la survie. Les pensées sont dangereuses, tout autant que le langage, en ce qu'ils donneraient corps à la réalité de l'état de grossesse, à cette vie en route, à la potentialité d'un enfant. Dans la violence de cette confrontation, la pensée se trouve paralysée, dans le même mouvement où le temps est suspendu: «Ce n'était pas la peine de nommer ce que j'avais décidé de faire disparaître. » Le Je s'interdit de nommer ce qui constitue sa réalité, il s'enferme alors dans un rapport paradoxal au langage et à la représentation.

Dans la négation par cette femme de son état de grossesse, le temps refusé attaque en retour l'inscription du sujet dans la chaîne temporelle; il met à mal le processus d'historisation, fondateur du Je (Piera Aulagnier, 1979,1984). Celui-ci s'en trouve amputé en retour, dans son impossibilité même de s'identifier, et de s'auto-représenter. L'établissement d'une continuité est bien la condition requise pour prendre place comme sujet dans une histoire généalogique. Pour ce faire, le Je doit s'assurer d'une certaine prévisibilité, anticiper un temps futur ${ }^{4}$.

\section{Interdit de représentation, faute et culpabilité}

Lors de cette grossesse, l'auteure met donc en œuvre un interdit d'autoreprésentation. Elle se condamne au silence, et dans ce mouvement conserve pardevers elle, et isole les éprouvés sensoriels et émotionnels dans toute leur densité, en une jouissance narcissique, phallique, mortifère ${ }^{5}$, dont la culpabilité constituera la clef de voûte.

«Il y a une semaine que j'ai commencé ce récit, sans aucune certitude de le poursuivre. Je voulais seulement vérifier mon désir d'écrire là-dessus. [...] je résistais sans pouvoir m'empêcher d'y penser. M'y abandonner me semblait effrayant. Mais je me disais aussi que je pourrais mourir sans avoir rien fait de cet événement. S'il y a une faute, c'était celle-là.» (p. 24).

La «faute » est liée à cette clôture que la mise en écriture est censée faire chuter. Cette mise en récit permettra à A. Ernaux, au terme de la narration d'écrire:

«J'ai effacé la seule culpabilité que j'aie jamais éprouvée à propos de cet évènement, qu'il me soit arrivé et que je n'en aie rien fait. Comme un don reçu et gaspillé. Car par-delà toutes les raisons sociales et psychologiques que je peux trouver à ce que 


\begin{abstract}
j'ai vécu, il en est une dont je suis sûre plus que tout: les choses me sont arrivées pour que j'en rende compte. Et le véritable but de ma vie est peut-être seulement celui-ci : que mon corps, mes sensations et mes pensées deviennent de l'écriture, c'est-à-dire quelque chose d'intelligible et de général, mon existence complètement dissoute dans la tête et la vie des autres » (p. 112).
\end{abstract}

Dans ce paragraphe qui compte parmi les dernières pages du livre, on retrouve le même mouvement, les mêmes mots que ceux de la citation précédente (posée tôt dans l'écriture, p. 24), soit la faute, et la culpabilité, et la réitération du n' «en avoir rien fait». Si l'écriture de cette tranche de vie entraîne à nouveau un écoulement du temps, et permet au Je de réintégrer ces éléments clivés, en renonçant à la part de jouissance préservée dans le clivage, c'est précisément dans la socialisation de cette expérience : avoir pu en «faire quelque chose». L'écriture même produit de la liaison dans cette mise en mots et en images pour d'autres. L'auteure témoigne que ces territoires psychiques n'étaient précédemment, que «sensations violentes », que « saisissement sans images ni pensées »; la nomination elle-même était hors de portée.

\title{
Appropriation, exhibition et sidération
}

Au-delà du seul récit de l'avortement, c'est toute sa vie dont Annie Ernaux rend compte au fil de ses publications. Par ce passage de l'écriture et dans une revendication identitaire à être «écrivain », elle tente de s'extraire de l'engluement dans l'expérience, de l'historiser, de la réintégrer dans une chaîne temporelle, et subjective. Exclusivement autobiographique, l'écriture est érigée en mode d'appropriation. Le statut d'écrivain combine alors les figures du «héraut» et celle du «héros». Au travers de la mise en récit, elle devient le héraut de sa geste héroïque $^{6}$. Avec ce récit autobiographique («L'événement»), comme avec l'ensemble de l'écriture de cette auteure, on se trouve en présence d'un double mouvement: une dynamique consiste bien dans cette visée d'humanisation de l'expérience; une autre en une (nouvelle) visée de dépossession. Cette dernière trouve à se jouer dans l'exhibition qui effracte et efface les frontières, détruit l'intime, et se donne comme agir violent.

Concernant l'humanisation de l'expérience, les sentiments de faute et de culpabilité dont l'auteure fait état, exigent de lui qu'il les transforme en «quelque chose d'intelligible et de général» pour les autres. Une place est alors revendiquée : «le véritable but de ma (sa) vie», celle d'en faire de l'écriture : «que mon corps, mes sensations et mes pensées deviennent de l'écriture ». L'auteure aspire non pas à la propriété, de ce/son corps, de ces/ses pensées, de ces/ses sensations et de ces/ses affects, mais à une mise en partage, de sorte qu'ils deviennent «intelligibles» pour d'autres, qu'ils rejoignent «la généralité » de l'expérience humaine. On se trouve là sur un registre de revendication: ce que le Je a expérimenté comme préjudice, il en réclame la reconnaissance par d'autres, et la 
violence en partage pour d'autres. Dès lors se profile un nouveau voilement. L'exhibition le dispute à des agirs de haine.

L'écrit a donc aussi pour fonction de tenter de «rendre » à l'autre la violence vécue - subie - l'autre étant ici parfaitement indéfini, et se confondant avec socius; cette mère société, qui n'a pas su protéger le sujet des épreuves et de la déshérence. Une des manières de composer avec le traumatisme consiste à transférer sur l'autre une part de cette violence vécue-subie, de la lui mettre littéralement (littérairement) sous les yeux. Dans son écrit, Annie Ernaux, énonce ce mouvement en clair.

«Le désir qui me poussait à dire ma situation ne tenait compte ni des idées ni des jugements possibles de ceux à qui je me confiais. Dans l'impuissance dans laquelle je me trouvais, c'était un acte dont les conséquences m'étaient indifférentes, par lequel j'essayais d'entraîner l'interlocuteur dans la vision effarée du réel. » (p. 57).

L'auteure fait état de la nécessité dans laquelle elle va se trouver (au moment des faits) d' «effarer», d' «égarer» l'autre, par la vision, de le sidérer dans l'exhibition de cette expérience traumatique.

«Plus tard, en mars, j'ai revu [...] l'étudiant qui m'avait accompagnée jusqu'au bus, quand je m'étais rendue pour la première fois chez le gynécologue. [...] Avec des détours, je lui ai fait comprendre que j'avais eu un avortement. C'était peut-être par haine de classe, pour défier ce fils de directeur d'usine, parlant des ouvriers comme d'un autre monde, ou par orgueil. Quand il a saisi le sens de mes paroles, il a cessé de bouger, ses yeux dilatés sur moi, sidéré par une scène invisible, en proie à une fascination que je retrouve toujours chez les hommes dans mon souvenir [...]. Il répétait, égaré, chapeau, ma vieille chapeau !» (p. 109)

Dans la narration de la rencontre avec cet étudiant en médecine, l'auteure inclura une note de bas de page visant à souligner la violence des «hommes ». Au travers de ce mouvement, elle dénie alors sa propre violence, celle qu'elle avait alors agi auprès de «ce fils de directeur d'usine»(et du lecteur). La note se présente comme une «association libre», autour de cette fascination des hommes, face aux mystères du féminin et aux territoires de l'archaïque (où les mystères de la maternité voisinent avec le pouvoir de mort et de dévoration). L'auteure tente ici de recouvrir la puissance de mort à l'œuvre dans l'expérience d'avortement, par «la fascination» des hommes pour les femmes. Elle efface la question de cette puissance de mort au moment où elle affleure, la retournant dans l'image de 
meurtres perpétrés par un homme sur des femmes et des fœtus. La violence «agie» est transformée en violence «subie». L'impensable pouvoir de mise à mort du fœtus est ainsi attribué à un personnage masculin tout droit sorti de la littérature; la confrontation à l'exorbitant pouvoir du maternel et à la confusion entre vie et mort qui s'y précipite, est escamotée dans une assimilation à une position victimaire. L'association de pensée de la narratrice dit ceci:

«Et que j' ai reconnue aussitôt, immense, chez John Irving, dans son roman L'œuvre de Dieu, la part du Diable. Sous le masque d'un personnage, il regarde mourir les femmes dans des avortements clandestins atroces, puis les avorte proprement dans une clinique modèle ou élève l'enfant qu'elles abandonnent après l'accouchement. Rêve de matrice et de sang où il s'adjuge et réglemente le pouvoir de vie et de mort des femmes.» (p. 109).

Il est d'autres moments dans l'écriture, où l'on perçoit comment une identification à «la femme victime de la violence» des hommes (ces derniers sont alors instantanément assimilés à des dominants), vient soutenir le mouvement d'effondrement psychique que le travail de remémoration de ce carrefour identificatoire (de l'avortement) fait redouter à son auteure. Les mouvements de revendications «féministes » et «politiques » viennent jouer les étais au fil de ce travail d'humanisation du traumatisme.

«Et si je ne vais pas au bout de la relation de cette expérience, je contribue à obscurcir la réalité des femmes et je me range du côté de la domination masculine du monde.» (p. 53).

\section{La haine différenciatrice; l'identification refusée à la mère}

Le mouvement de haine adressé à «l'homme ${ }^{7}$ » sert de rempart au Je, et le constitue paradoxalement dans une différence: les hommes contre les femmes, les dominants contre les dominés. Il y a danger en effet à se rapprocher des identifications refusées à la mère.

«J'ai su que j'avais perdu dans la nuit le corps que j'avais depuis l'adolescence, avec son sexe vivant et secret, qui avait absorbé celui de l'homme sans en être changé — rendu plus vivant et plus secret encore. J'avais un sexe exhibé, écartelé, un ventre raclé, ouvert à l'extérieur. Un corps semblable à celui de ma mère. » (p. 98).

Au travers de cette expérience de grossesse et d'avortement, l'auteure se retrouve dans une obligation de se confronter à ses identifications féminines et maternelles. Elle est alors aux prises avec la figure d'une mère archaïque qui vient 
la menacer d'anéantissement. Retrouver en soi l'analogue du corps de la mère («Un corps semblable à celui de ma mère ») va s'effectuer au prix de la destruction de la dimension de l'intimité.

Le sexe féminin en tant que lieu de l'intime devient le lieu du retournement («ouvert à l'extérieur»). D'une appropriation corporelle de son sexe comme un lieu «vivant et secret», et d'une sexualité que l'auteure désigne comme l'ayant rendue plus vivant(e) et plus secret(e) encore, lui permettant de sortir du contrôle maternel, le bouleversement de l'avortement transforme ce sexe en lieu de l'exhibition («un sexe exhibé, écartelé»), objet de sévices («écartelé, raclé»), sous la domination et le regard de l'autre. Aussi pour nier la possible assimilation avec cette mère interne, la jeune femme d'alors se réfugiera dans le refus: celui de consentir à participer d'une mêmeté. Ce qui est habituellement source et créateur d'intimité va être retourné en exhibition ${ }^{8}$. De cela l'écriture autobiographique témoigne également.

La dimension de l'intime est expulsée. S'il n'y a pas de place pour une imago positive de la mère (une imago suffisamment bienveillante) dans la psyché de l'auteure, elle se trouve dans la nécessité de s'inventer un arrimage identitaire réparateur et générateur d'intériorité. Si l'intimité est fantasmée comme étant l'objet de la curiosité maternelle — la mère surveille la sexualité de sa fille ${ }^{9}$ source d'une possible jouissance maternelle, l'expérience d'avortement et la confusion des corps (corps d'adolescente et «corps semblable à celui de ma mère »), font éprouver au Je la menace de perdre ce qui dans la sexualité qui lui avait permis la constitution d'un territoire «secret», la préservation d'un espace psychique et corporel, hors de portée de l'emprise d'une mère archaïque. Le Je se trouve menacé d'intrusion et doit s'inventer une nouvelle posture qui le situe hors de ces zones confusionnantes.

\section{Le meurtre de la mère : trauma, confusion et nouveau point d'origine}

«Je suis parvenue à l'image de la chambre. Elle excède l'analyse. Je ne peux que m'immerger en elle. Il me semble que cette femme qui s'active entre mes jambes qui introduit le spéculum, me fait naître.

J'ai tué ma mère en moi à ce moment-là. » (p. 77).

Ces phrases cinglantes condensent la terrible confusion dans laquelle le sujet va se précipiter. C'est à l'instant du geste meurtrier du fœtus qu' Annie Ernaux arrime une nouvelle origine pour elle. C'est du geste intrusif pratiqué par une femme dans son sexe qu'elle se fait naître. Une femme tue le maternel en elle en tuant le fœetus dans sa matrice - Au cours de la narration l'auteure réitérera l'énoncé de ce collage identificatoire à ce moment confusionnant, allant jusqu'à rendre hommage à la femme qui l'a avorté (et souhaiter lui dédicacer l'ouvrage). Pour le Je, c'est l'assimilation au moment sidérant qui l'«arrache» à la mère. 
« Je n'ai jamais revu $\mathrm{M}^{\mathrm{me}} \mathrm{P}$.-R. Je n'ai jamais cessé de penser à elle. Sans le savoir cette femme $[\ldots]$ m'a arrachée à ma mère et m'a jetée dans le monde. C'est à elle que je devrais dédicacer ce livre.» (p. 111).

On assiste au travers de ces quelques lignes à la validation d'un fantasme d'auto-engendrement. Pour le Je il y a lieu de se faire naître et que la mère meure. Il y faut de la mort. L'acte transgressif de l'avortement rejoint alors le fantasme du «matricide», et précipite celui d'un auto-engendrement, dans la mort du fœtus.

«Je ne me sentais pas différente des femmes de la salle voisine ${ }^{10}$ [...] Dans les toilettes de la cité universitaire j'avais accouché d'une vie et d'une mort en même temps. Je me sentais pour la première fois, prise dans une chaîne par où passaient les générations.» (p. 103).

Valse folle des places: «J'avais accouché d'une vie» (la sienne), «d'une $[\ldots]$ mort» (celle du fœtus, celle de la mère) «J'ai tué ma mère en moi à ce momentlà.». Cet instant confusionne, en ses multiples équivalences: je tue l'enfant audedans de moi, je tue la mère en moi. Renversement chaotique des places (Qui est la mère, qui est l'enfant ?), accomplissement d'un agir de haine... Il ne saurait y avoir d'espace pour une mère et son enfant, pour elle et sa mère. La configuration aliénante réclame de la violence meurtrière, assimilé enfin à une séparation.

Dans la même phrase est aussi énoncé le paradoxe de se sentir «prise dans une chaîne par où passaient les générations », au moment même de la destruction de la filiation, destruction psychique des ascendants et destruction psychique des descendants.

C'est à partir de ce point traumatique que le Je va «s'originer» désormais.

«Pendant des années, la nuit du 20 au 21 janvier a été un anniversaire.» (p. 111).

\section{L'écriture autobiographique : exhiber et détruire l'intime}

L'écriture autobiographique apparaît pour le Je comme une issue viable face à la confusion. Tout ce qui pourrait relever de l'intimité, constituer de l'intimité, est transformé en matériel d'écriture, réalisant un double mouvement de reconnaissance et d'expulsion. L'expérience est reconnue par le Je, mais soustraite à la «toute vision » du surmoi archaïque. Si le sujet se fait naître au moment sidérant, il s'assimile aussi identitairement au mouvement du passage entre éprouvé intime et exhibition publique dans l'écriture: «Et le véritable but de ma vie est peut-être seulement celui-ci : que mon corps, mes sensations et mes pensées deviennent de l'écriture, c'est-à-dire quelque chose d'intelligible et de général, mon existence complètement dissoute dans la tête et la vie des autres» (p. 112). Cette incessante 
transformation-expulsion de l'intime en matériel d'écriture, confère au Je une reconnaissance au travers du statut d'écrivain, et lui fournissant une seconde enveloppe, véritable matrice sociale. C'est du socius qu'Annie Ernaux réclame un nouveau statut identitaire, public celui-là.

Le lien entre la position d'écrivain et le traumatisme de «l'événement» (ce point d'origine auquel le sujet s'assimile) nous est aussi signifié par l'auteure ellemême. Faisant référence à cette chambre du logement de l'aide-soignante qui l'a avortée, et s'interrogeant sur l'énigmatique de la situation, elle écrit :

«(Ce n'était donc pas le malheur. Ce que c'était vraiment serait peut-être à chercher dans la nécessité que j'ai eue de m'imaginer à nouveau dans cette chambre, ce dimanche-là, pour écrire, Les armoires vides, huit ans après. Dans le désir de faire tenir, dans ce dimanche et dans cette chambre, toute ma vie jusqu'à vingt ans.)» (p. 88).

Dans ce témoignage, elle ré-énonce comment le moment traumatique, l'arrimage au fœticide, vient agglomérer, condenser «toute (sa) vie jusqu'à vingt ans ». Les armoires vides, c'est le titre qu'Annie Ernaux donnera à son premier ouvrage publié, (1974), celui qui l'établira comme écrivain. Ce premier roman s'ouvre du reste sur la description de la scène d'avortement. Cette scène constitue donc un véritable fil rouge autour duquel s'est axée la position d'écrivain.

Ce mouvement de violence dessine une configuration telle que: «moi ou l'autre », (elle ou l'enfant - elle ou la mère) qui procède d'une configuration familiale aliénante, d'un trouble de la filiation et d'un trait paranoïaque ${ }^{11}$. C'est de l'aliénation ${ }^{12}$ de la psyché de l'enfant par la psyché maternelle, dont le sujet va devoir s'extraire. Le mouvement qui mène l'auteure de «l'événement » à énoncer ce matricide psychique (tuer le tyran interne), est aussi appel à l'homme à ce carrefour, même si cet appel le convoque à l'endroit de la violence meurtrière (des femmes et des fotus). On a vu que cette convocation vient en effet voiler la violence du maternel archaïque, et jouer comme autorisation à une première mise en pensée de cette configuration chaotique. Ce mouvement est donc coloré d'une dynamique œdipienne, même si elle signe simultanément l'échec partiel de la mise en place du père pour faire barrage à la mère archaïque. La scène publique, la notoriété de l'écrivain promeuvent le patronyme paternel, à partir duquel cette femme se signifie.

\section{Le statut d'exception, «enveloppe » pour le Je}

Le mouvement de destruction de l'intime, de cette catégorie habituellement structurante pour les sujets, et l'expulsion, via l'écriture (toute expérience, tout éprouvé devenant publics et publiables) témoigne d'une configuration en emprise. À défaut d'avoir été autorisé à se construire un territoire préservé de l'intrusion de l'autre, c'est dans le regard de cet autre que le Je se met à rechercher les effets du 
trouble. Il se met en position de «squatter» la psyché de l'autre, pour mesurer les effets produits, et/ou les exporter et s'en débarrasser. Les éléments qui vont demeurer déliés pour le Je trouvent à se lier dans la pensée et/ou le corps du lecteur.

Il s'agit dès lors de se soumettre au regard de l'autre, et de n'avoir de cesse de vérifier de n'en être pas détruit: étonnante manière de composer avec un surmoi féroce et tyrannique, qui ne cesse de pousser à la jouissance. Dans un autre ouvrage, La honte (1997), en quatrième de couverture, on trouve cet énoncé qui indique, on en peut plus clairement, cette recherche de découverte d'une posture d'écriture «dont il (lui) soit ensuite impossible de parler, qui rende le regard d'autrui insoutenable».

«J'ai toujours eu envie d'écrire des livres dont il me soit ensuite impossible de parler, qui rendent le regard d'autrui insoutenable. Mais quelle honte pourrait m'apporter l'écriture d'un livre qui soit à la hauteur de ce que j'ai éprouvé dans ma douzième année. »

Ces propos de l'écrivain illustrent au mieux le mouvement que l'écriture autobiographique instaure. L'exhibition se fait violence imposée à l'autre comme un rendu des effractions vécues, de l'effacement (de la perte) de l'intimité. Le Je n'ayant pu s'éprouver suffisamment respecté à l'endroit de son intimité, il va se donner à voir tout entier, en mes moindres replis. L'acte vaut alors comme énoncé : Si mon intimité vous intéresse (intéresse l'autre maternel), alors regardez. Peutêtre ainsi apprendrais-je à saisir la limite qu'indique votre trouble — discours interne que le Je adresse à ses persécuteurs (à la figure de la mère archaïque), dans une quête de limite à la confusion. Vers la fin de la narration de «l'événement», cette même confusion fait dire à l'auteure: «[...] j'ai remplacé mes lunettes par des lentilles dont l'ajustement sur l'œil me paraît aussi difficile et hasardeux que celui du diaphragme au fond du vagin. » (p. 111) — par où s'entend la confusion entre le regard et l'intimité du sexe, œil et vagin confondus. Dans cet énoncé se dessine aussi la volonté de se maintenir en un lieu de violence où demeure centrale la visée de sidération de l'autre: là où les images crues sont livrées, exhibées, à charge pour le lecteur de réaliser le travail de métabolisation de ces évènements et de ces tranches de vie, ou de s'y effarer.

Travaillant sur la notion d'emprise, et sur ses manifestations dans la littérature, Alain Ferrant (2001) écrit à propos de Ferdinand Céline: «Céline s'efforce [...] de se loger dans la tête du lecteur afin de se confondre avec lui» (p. 172). Ferrant déconstruit le style célinien pour indiquer par quels mouvements d'écriture cette visée se réalise, déstabilisant systématiquement tous repères susceptibles de permettre au lecteur de demeurer tel (et de conserver une distance suffisante au travers de sa posture habituelle de lecteur). Annie Ernaux prétend explicitement à cette même visée de dissolution dans l'autre. Ce faisant elle dépose dans le corps 
du lecteur les éléments traumatiques. Ici le style tente de dupliquer les sensations et les image, au plus près de leur pouvoir de sidération initial. L'écrivain fait en sorte que les sensations acquièrent pour le lecteur la même portée qu'elles ont eue pour elle. Elle se livre ainsi à la description de scènes au plus près de leur vécu, dans une écriture d'autant plus crue que la pensée de l'auteure a été paralysée au moment des faits, et qu'elle en reconduit le mouvement.

\section{Identifications, classes sociales et transgression}

Il est donc des situations traumatiques qui éjectent le Je de ses repères identificatoires. Il s'agit alors de véritables situations-carrefours. Ce qui concourt à faire de «l'événement» un véritable traumatisme, c'est la sursaturation signifiante qu'il entraîne pour le Je. L'auteure témoigne, comment dans cette expérience viennent se précipiter l'ensemble de ses repères identificatoires antérieurs. Elle convoque notamment dans son propos le vacillement dans lequel elle se trouvait alors, relativement à ce qu'elle nomme sa «classe sociale».

«J'établissais confusément un lien entre ma classe sociale d'origine et ce qui m'arrivait. Première à faire des études supérieures dans une famille d'ouvriers et de petits commerçants, j'avais échappé à l'usine et au comptoir. Mais, ni le bac, ni la licence de lettres n'avaient réussi à détourner la fatalité de la transmission d'une pauvreté dont la fille enceinte était, au même titre que l'alcoolique, l'emblème. J'étais rattrapée par le cul et ce qui poussait en moi, c'était, d'une certaine manière, l'échec social. » (p. 29).

Soulignons ici que l'un des ouvrages qui a largement contribué à la notoriété de cette écrivain (prix Renaudot 1984) a pour titre La place (1983). Cet ouvrage témoigne du cheminement social de l'auteure, qui «fille d'ouvrier, devenu petit commerçant », se retrouve seule de sa famille (et de son entourage) à entreprendre des études supérieures. Le livre décrit l'intériorisation des normes sociales et les incidences des appartenances de classes. Cet ouvrage a fait l'objet d'une reprise théorisante, de Vincent de Gaulejac, au titre d'illustration pour ses hypothèses concernant «La névrose de classe». Cette recherche est bien entendue connue d'Annie Ernaux, qui a elle-même signé quelques mots dans l'ouvrage de V. de Gaulejac (1987). Elle considère les déterminants sociaux, les habitus (Bourdieu 1982) comme de toute première importance. À l'occasion d'une émission littéraire télévisuelle lors de la sortie de «L'événement», elle témoignera de son désintérêt pour la psychanalyse, mettant une nouvelle fois l'accent sur les déterminants sociologiques. Nous émettons l'hypothèse que les travaux de V. de Gaulejac qu'Annie Ernaux reprend à son compte, et l'insistance concernant cette dimension de classes sociales lui permet d'éviter une confrontation avec sa propre dynamique psychique. Elle préserve en cela le compromis identificatoire auquel elle est parvenue, notamment la place héroïque qu'un tel parcours lui octroie, et la place de «dichter», qu'elle compose. 


\section{«Hors temps », sacralisation et chaîne des générations}

À propos de la structure narrative de L'événement, soulignons que l'écriture nous conduit dès la première page, dans la salle d'attente d'un hôpital. C'est l'analogue entre deux situations qui réveille pour Annie Ernaux le souvenir enfoui de l'avortement. On est ici dans un effet de concordances, d'emboîtement. Une situation vient en réveiller une autre, et permettre de confronter à ce qui a été clivé, trente-six ans auparavant. Dans le décours de la narration, le lecteur est progressivement informé que l'auteure se trouve dans un service hospitalier pour chercher réponse à un test de dépistage du sida. Ce premier tableau, constitue le premier chapitre (quelques pages concises); il se conclut par le paragraphe suivant:

«Je me suis rendu compte que j'avais vécu ce moment à Lariboisière de la même façon que l'attente du verdict du docteur N., en 1963. Ma vie se situe donc entre la méthode Ogino et le préservatif à un franc dans les distributeurs. C'est une bonne façon de la mesurer, plus sûre que d'autres, même.» (p. 16).

En ces lignes Annie Ernaux indique comment sa vie s'inscrit entre deux moments, deux passages en hôpital, qui convoquent les mêmes questions identitaires et fondatrices: la sexualité et la fécondité s'y conjuguent avec la mort et le refus. Ces situations apparaissent donc comme des ponctuations, des bornages d'un temps psychique, un temps hors temps, que le Je va transformer en point d'origine, où il vient se perdre, en se coupant des identifications qui l'insèrent dans la chaîne des générations.

\section{L'appel au sacré sur les franges de l'abjection}

Lorsque le Je a vécu des situations susceptibles de le détruire, il revendique le dédommagement du préjudice. Une des exigences fréquentes est alors celle de l'octroi du statut d'exception. L'expérience d'Annie Ernaux indique une des composantes de cet autre mouvement de retournement à l'œuvre: il va s'agir de sacraliser, ce qui pourrait entraîner l'auteure à se vivre comme hors humanité, et courir le risque d'une déliaison majeure d'avec la communauté humaine. L'expérience qu'elle va s'efforcer de sacraliser l'amène à s'assimiler aux exclus, auxquels elle-même octroie un statut héroïque, à partir de l'idée qu'ils sont capables de se tenir debout, désarrimés du groupe. Une nouvelle fois on se trouve en présence d'une configuration qui implique l'éviction de la temporalité au travers de la sacralisation. Le sacré vient produire un suspens de la temporalité. Le mouvement de l'écriture en ce sens, parachève le mouvement, inscrivant l'expérience dans le registre de la «vérité — toute vérité étant dès lors «bonne à dire» contrairement au dicton populaire. 
«Il se peut qu'un tel récit provoque de l'irritation, ou de la répulsion, soit taxé de mauvais goût. D'avoir vécu une chose, quelle qu'elle soit donne le droit imprescriptible de l'écrire. Il n'y a pas de vérité inférieure. » (p. 53).

Ce qu'ambitionne Annie Ernaux c'est précisément d'inscrire son expérience comme une expérience «sacrée». En plaçant cette expérience sous le sceau du «sacré », un sacré de «transgression», il s'agit pour le Je de se couper de ses origines, de rompre une filiation vécue comme emprise aliénante.

\section{L'éprouvé traumatique et la dissolution du Je}

Au travers de cette expérience d'avortement, Annie Ernaux témoigne de la part de confusion, d'indécidable ${ }^{13}$, liée à ces vécus «extrêmes »; indécidable quant à savoir si l'expérience relève du registre de «l'horreur» ou de celui de «la beauté », si elle déloge le Je de sa place d'humain et le réfère à l'abjection, ou si elle lui confère un statut d' «exception», une place de héros.

«Je marchais dans les rues avec le secret de cette nuit [...] dans mon corps, comme une chose sacrée. Je ne savais pas si j'avais été au bout de l'horreur ou de la beauté. J'éprouvais de la fierté. Sans doute la même que les navigateurs solitaires, les drogués et les voleurs, celle d'être allé jusqu'où les autres n'envisageront jamais d'aller. C'est sans doute quelque chose de cette fierté qui m'a fait écrire ce récit. » (p. 107).

Une telle narration permet d'épingler un ensemble de mouvements configurés selon la figure clef du retournement, au titre desquels celui qui transfigure l'expérience confusionnante en «une chose sacrée ». La fierté — prime narcissique à laquelle va prétendre le Je — signe l'identification imaginaire censée être conférée par ces incursions sur ces territoires expérientiels, là «où les autres n'envisageront jamais d'aller». En ces lieux pointent la transgression et la jouissance corrélée, dans leurs versants mortifères - transgression explicitement référée au travers de l'appel aux figures des drogués, et des voleurs. C'est ce même mouvement d'arrimage narcissique qui permet au Je d'ignorer ce qu'il joue de sa déshérence, lors même qu'il est entièrement assimilé à son éprouvé, dans le clivage.

Ces vécus expérientiels «exceptionnels» procèdent donc d'un mouvement paradoxal, de différenciation/indifférenciation, d'identification à la figure grandiose et désincarnée du héros. Annie Ernaux témoigne de cette précipitation hors des limites, sachant que c'est alors l'expérience d'écriture qu'elle met en place de bordure, et qui fait barrage à la tentation de dissolution énoncée. Une telle position d'écriture constitue précisément l'opposé d'un «hors limite», puisque produisant une trace, et faisant le renom de son auteure, en promouvant son patronyme. Le style qui «signe» l'auteure participe de ce même mouvement. 
Le Je s'éprouve vivant, corrélativement à la menace qu'il a éprouvé de «s'y perdre». Les confusions qu'il a affrontées lui permettent d'imaginer ne pas prendre place dans le lien générationnel; l'expérience est ainsi placée sous le sceau du «sacrifice ${ }^{14} »$. Ces expériences «limites» mettent à mal les frontières à partir desquelles le Je circonscrit sa résidence, notamment au travers de la destruction de la pensée; celles-ci se trouvant aspirées dans la béance créée par le traumatisme.

Le récit de l'avortement, relaté de façon crue, «factuelle», n'épargne au lecteur aucun détail. L'appel au «sacré», énoncé précisément sur ces franges de l'abjection, vient noyer, ce qui pourrait apparaître de la vie pulsionnelle et de l'organisation libidinale, et serait susceptible d'entraîner un travail de réflexivité, voire une perlaboration.

La mise en récit permet toutefois à A. Ernaux de s'extraire de la sidération, du temps aggloméré (ce temps «hors temps») et, dans la mise en partage avec le plus grand nombre (par le biais de l'édition), remet en jeu la sidération qu'elle sait possible de faire éprouver à l'autre. À défaut de faire elle-même de «l'événement » l'objet d'une élaboration psychique, cet évènement acquiert un statut «culturel», via la notoriété de l'écrivain. Elle tente ainsi de maintenir la satisfaction narcissique octroyée par le franchissement incessant d'une limite : celle de la destruction revendiquée de l'intime, dans la dépossession qu'en réalise le récit autobiographique. Ce franchissement lui fait quitter un statut identificatoire mal différencié d'avec l'autre maternel, et en acquérir un nouveau à partir de la transgression.

Cette transgression vaut comme fondement narcissique — traumatique, pour la rupture qu'elle génère. Ce refus (refus de s'identifier à son sexe dans la génération) se double de celui de tout travail de pensée élaboratif aux fins de conserver intacte la charge émotionnelle et sensorielle, qui vaut alors comme sceau de l'autoengendrement.

\section{Traumatisme, transgression et auto-fondation La revendication hérö̈que, comme issue au traumatisme}

En introduction à cet article, nous avons souligné combien la narration de cet événement par Annie Ernaux, en son condensé, avait valeur paradigmatique de ces mouvements de revendication identitaire à l'endroit du traumatisme, lors de l'entrée dans l'âge adulte.

Cet écrit donne en effet une grande lisibilité au destin de ces traumatismes qui signent cette entrée aux yeux du Je. Lors de ces remaniements identificatoires, le Je met en acte son appétence traumatophilique (Guillaumin, 1985). Il s'arrime alors au traumatisme et/ou à l'acte transgressif comme à un point d'origine en un mouvement d'auto-fondation, d'auto-engendrement. Le Je peut ainsi choisir de se saisir du traumatisme pour l'ériger en moment fondateur.

Cette manière d'utiliser le traumatisme constitue certes une manière de l'intégrer, mais en s'y soumettant et en s'y perdant. Se met alors en place une nouvelle excitation qu'il va s'agir d'alimenter sans cesse. Le moment traumatique 
se transforme en point d'origine imaginaire. Cette prévalence de l'imaginaire dans lequel le sujet s'engouffre le désarrime de la chaîne symbolique et générationnelle. L'expérience traumatique devient son origine mystérieuse. Le sujet est seul à savoir qu'il est «né » à ce moment-là, ce qui dissout pour lui le lien d'inclusion dans une chaîne généalogique et signifiante, et le précipite hors de la temporalité. Le récit d'Annie Ernaux permet d'entendre finement combien la solitude, dans laquelle se déroulent ces expériences (de traumatisme et de vécu transgressif) potentialise l'exclusion. Cette exclusion se donne alors comme la glorification du hors la loi et comme clôture auto-fondatrice. La visée de complétude recouvre ce qui dans le traumatisme ravive précisément la marque de la castration, et qui devient l'objet du refus.

L'expérience traumatique est conservée dans sa charge d'excitation (sa sensorialité et sa sursaturation signifiante) valant paradoxalement contre l'excitation incestueuse-incestuelle. Le traumatisme est cadré par le Je comme signant une séparation jamais assurée, comme un refus identificatoire par crainte d'une aliénation totale à l'emprise maternelle. L'issue est alors la revendication d'une position héroïque comme dédommagement d'un préjudice subi.

\section{Sidérer l'autre comme preuve de la traversée du carrefour identitaire}

Corrélée à ce mouvement de prétention identitaire, l'autre dynamique d'utilisation du traumatisme consiste à faire éprouver à l'autre une part de la sidération par soi-même éprouvée, et d'en jouir en retour. Le Je tente d'évacuer projectivement dans l'autre, la violence à laquelle il s'est trouvé confronté, d'exporter ce qui a fait trou dans sa psyché. C'est alors dans la sidération éprouvée par l'autre que le Je reconnaît qu'il a survécu, qu'il a réussi à supporter l'épreuve (recherchée et/ou subie). Un tel mouvement de sidération aperçu chez l'autre devient la preuve de son propre statut héroïque. Ce mouvement de reprise à l'identique (de M'Uzan, 1969) ne permet pas de déboîter de la position construite par le Je. Il participe tout au contraire au renforcement de cette position, et oblitère tout travail de reprise élaborative.

Il s'agit d'amener l'autre au point énigmatique que le Je a dû affronter. Dans le même temps où il est disqualifié ${ }^{15}$, l'autre est aussi recherché dans sa potentialité thérapeutique. Dans un éprouvé solitaire que le sujet s'efforce de préserver, le traumatisme lié à la transgression, vient se configurer contre la pensée, et établir la prévalence de l'auto-engendrement mortifère. Le Je cherche toutefois à passer par l'autre (par les autres) dans l'espoir, qu'il en sera un à même de ne pas céder à la fascination de l'archaïque, qu'il en sera un à même de l'aider à dépasser ses identifications aliénantes via un travail de reprise historisant de sa trajectoire.

georges gaillard institut de psychologie université lumière lyon 2 lyon, france georges.gaillard@wanadoo.fr 


\section{Notes}

1. Annie Ernaux (2000), L'événement, Paris, Gallimard, p. 112.

2. P. Aulagnier (1967), Le «désir de savoir» dans ses rapports à la transgression — in L'inconscient, $\mathrm{n}^{\circ}$ 1, repris dans Un interprète en quête de sens, Paris, Ramsay, (1986), p.156.

3. Parmi les travaux qui mettent l'accent sur ces mouvements de séparation qu'opèrent les «initiations traditionnelles, dans leur lien avec le traumatisme citons Tobie Nathan 1987 (Traumatisme, identification et mémoire - in Aïn J. et al., Adolescences, Miroir des âges de la vie, Toulouse, Privat p. 147-158), et Martine Stassart 1996, (Anthropologie de l'adolescence in Cahiers du C.E.P., $\mathrm{n}^{\circ}$ 7, p. 22-23).

4. «La tâche du Je sera de transformer ces documents fragmentaires» (les traces et les récits de son histoire) «en une construction historique qui apporte à l'auteure et à ses interlocuteurs le sentiment d'une continuité temporelle.» (P. Aulagnier 1984, L'apprenti historien et le maître sorcier, p. 196).

5. Selon la spécification que propose Paul Laurent Assoun (1994 a), La psychanalyse à l'épreuve du pouvoir, in Analyse et réflexion sur le pouvoir, Vol. II, Ouvrage collectif, Ellipses, Paris 1994, p. 59-71.

6. Freud a souligné (1912) comment la figure du «héraut » jouxte, et s'intrique à celle du «héros».

7. De la violence à rendre il en est également explicitement question à propos de l'hospitalisation qu'elle nommera une «prise en charge punitive de l'Hôtel Dieu », et d'un interne de garde dont elle dit éprouver le désir de donner son nom en pâture, à ses lecteurs. «(Si j'avais connu le nom de cet interne de garde pendant la nuit du 20 au $1^{\text {er }}$ janvier 64 et que je m'en souvienne, je ne pourrais m'empêcher maintenant de l'écrire ici. Mais il s'agirait d'une vengeance inutile et injuste dans la mesure ou son comportement ne devait constituer que le spécimen d'une pratique générale).» - Annie Ernaux (2000), op. cit., p. 100-101.

8. Durant ce temps de l'avortement, l'exhibition et l'intrusion sont parfois littérales : «Dans la salle d'opération, j'ai été nue, les jambes relevées et sanglées dans des étriers sous la lumière violente.» (p. 77).

9. Parmi les rares occurrences de la figure de la mère, dans le texte de L'événement, la première concerne la position de contrôle: «Au week-end de la Toussaint, je suis retournée comme d'habitude chez mes parents. J'avais peur que ma mère ne m'interroge sur mon retard. J'étais sûre qu'elle surveillait mes slips tous les mois en triant le linge que je lui apportais à laver.» (p. 19).

10. Il s'agit du service de gynécologie obstétrique de l'Hôtel Dieu, qui ne semble pas différencié du service de maternité. Les femmes de la salle voisine sont donc les jeunes mères qui viennent d'accoucher.

11. Il nous apparaît qu'une configuration relationnelle dans laquelle se met en place une violence de «moi ou l'autre » si elle procède d'une configuration familiale aliénante, d'un trouble de la filiation et d'un trait paranoïaque, ne saurait toutefois prétendre à un statut fondateur pour tous les sujets, — contrairement à la proposition de Jean Bergeret $(1984,2000)$ et à son concept de «violence fondamentale», auquel l'expression «moi ou l'autre» fait écho.

12 Nous entendons ici le terme «d'aliénation» comme catégorie structurelle, selon les propositions métapsychologiques de Piera Aulagnier (1984).

13. «Quelles que soient les théories Freudiennes du traumatisme on s'apercevra que toutes s'inscrivent sous cette forme fondamentale de l'indécidabilité subjective qui constitue le paradigme de toute situation et de toute construction traumatique. » Bernard Duez, 1999, p. 5-6.

14. «Nous sommes toutes les deux dans ma chambre. Je suis assise sur le lit avec le fotus entre les jambes. Nous ne savons pas quoi faire. Je dis à $\mathrm{O}$. qu'il faut couper le cordon. Elle prend une paire de ciseaux, nous ne savons pas à quel endroit il faut couper, mais elle le fait. Nous regardons le corps minuscule, avec une grosse tête, sous les paupières transparentes les yeux font deux taches bleues. On dirait une poupée indienne. Nous regardons le sexe. Il nous semble voir un début de pénis. Ainsi j'ai été capable de fabriquer cela. O. s'assoit sur le tabouret, elle pleure. Nous pleurons silencieusement. C'est une scène sans nom, la vie la mort en même temps. Une scène de sacrifice.» (Annie Ernaux, op. cit., p. 91. Le passage hors italique est souligné par nous).

15. Les «divans» connaissent bien ces mouvements de disqualification de cela même que les sujets recherchent « désespérément» - Par où se profile la part masochiste de telles configurations. 


\section{Bibliographie}

Assoun, P.L. (1999), Le préjudice et l'Idéal, Pour une clinique sociale du trauma, Paris, Anthropos, 253 p.

Aulagnier, P. (1975), La violence de l'interprétation. Du pictogramme à l'énoncé (cf. Castoriadis-Aulagnier P.), Paris, PUF, 363 p.

Aulagnier, P. (1984), L'apprenti historien et le maître sorcier, Paris, PUF, 276 p.

Declerck, P. (2001), Les naufragés, Paris, Plon, 455 p.

Doorey, R. (1988), Le désir de savoir, Paris, Denoël, 219 p.

Duiez, B. (1996), Psychopathologie de l'originaire et traitement de la figurabilité, in Kaës R. et al,, Souffrance et psychopathologie des liens institutionnels, Dunod, p. 161-203.

Ernaux, A. (1974), Les armoires vides, Paris, Gallimard, Folio, 182 p.

Ernaux, A. (1977), Ce qu'ils disent ou rien, Paris, Gallimard, Folio, 154 p.

Ernaux, A. (1983), La place, Paris, Gallimard, Folio, 114 p.

Ernaux, A. (1997), La honte, Paris, Gallimard, Folio, 142 p.

Ernaux, A. (2000), L'événement, Paris, Gallimard, 115 p.

Ernaux, A. (2001), Se perdre, Paris, Gallimard Folio, 384 p.

Ernaux, A. (2002), L'occupation, Paris, Gallimard Folio, 76 p.

Ferrant, A. (2001), Pulsion et liens d'emprise, Paris, Dunod, 205 p.

Freud, S. (1915), Pulsions et destin des pulsions, in Métapsychologie, trad. franç., Gallimard, 1968, p. 11-44.

Freud, S. (1920), Au delà du principe de plaisir, in Essais de psychanalyse, trad. franç. Paris, Payot, 1981, p. 41-115.

Freud, S. (1922), La tête de Méduse, in Résultats idées, problèmes II, trad. franç. Paris, PUF, 1985, p. 49-50

Gaillard, G. (2001), Identifications professionnelles, assignations institutionnelles et paralysies de la pensée, in Revue de psychothérapie psychanalytique de groupe, $\mathrm{n}^{\circ} 35$, Érès, p. 185-200.

Gaillard, G. (2004), De la répétition traumatique à la mise en pensée, in Revue de psychothérapie psychanalytique de groupe, $\mathrm{n}^{\circ}$ 42, Éditions Érès, p. 151-164

Gauléjac, V. De (1987), La Névrose de classe, Hommes et Groupe Éditeur, Paris, 308 p.

Guillaumin, J. (1992), L'emprise, la répétition aux frontières de l'appareil psychique, in Revue française de psychanalyse, tome LVI, De l'emprise à la perversion, p. 1485-1489.

Héritier, F. (2001), Inceste et substance. Edipe Allen, les autres et nous, Entretien avec J. André, in André J. (sous la direction de), Incestes, Paris, PUF, p. 91-133.

Janin, C. (1996, édition corrigée 1999), Figures et destins du traumatisme, Paris, PUF, 132 p.

Jeammet, P. (2000), L'énigme du masochisme, in André J. (sous la direction de), L'énigme du masochisme, Paris, PUF, p. 31-67.

Kaës, R. (1991), L'affinité du groupe du traumatisme psychique et de la crise, in Revue de Médecine Psychosomatique, $\mathrm{n}^{\circ} 27$ p. 77-89.

Kaës, R. (1992), Pacte dénégatif et alliances inconscientes, in Gruppo, nº 8, p. 117-118.

Lacan, J. (1972-1973), Le Séminaire livre XX, Encore, Paris, Seuil, 1975 b, 133 p.

Legendre, E P. (1985), L'inestimable objet de la transmission, Paris, Fayard, 408 p.

Mijolla-Mellor (de) S. (1992), Le plaisir de pensée, Paris, PUF, 413 p.

Pontalis, J.B. (1975), À partir du contre-transfert: le mort et le vif enlacés, in Nouvelle Revue française, 1975, $\mathrm{n}^{\circ} 12$, p. 73-87. 


\section{6}

Filigrane, printemps 2006

Racamier, P. C. (1995), L'inceste et l'incestuel, Paris, Les éditions du Collège, 250 p.

Roussillon, R. (1999 a), Agonie, clivage et symbolisation, Paris, PUF, 245 p.

Vasse, D. (1999), La dérision ou la joie, la question de la jouissance, Paris, Seuil, 319 p. 